\title{
Development of a Numerical Well Test Simulator for Polymer-surfactant Flooding and the Pressure Transient Response Study
}

\author{
Zhichun $\mathrm{Jia}^{1, \mathrm{a}}$, Daolun $\mathrm{Li}^{1, \mathrm{~b}}$ and Detang $\mathrm{Lu}^{1, \mathrm{C}^{*}}$ \\ ${ }^{1}$ University of Science and Technology of China, Hefei, Anhui, China. \\ a jzc@mail.ustc.edu.cn, ${ }^{b}$ Idaol@ustc.edu.cn, ${ }^{c}$ dtlu@ustc.edu.cn
}

Keywords: Well test analysis; Polymer-surfactant flooding; Numerical simulation; Enhanced oil recovery.

Abstract. The lack of well test interpretation techniques applicable to chemical flooding reservoirs has restricted the formation evaluation and project design in EOR (Enhanced Oil Recovery) production. In this study, a two-phase five-component well test model for polymer-surfactant flooding is proposed which considers the main physicochemical phenomena including polymer in-situ viscosity, permeability reduction, inaccessible pore volume, adsorption, phase behavior, capillary desaturation, and relative permeability. Base on PEBI gridding, a fully implicit numerical well test simulator is developed. The impact of polymer and surfactant injection on transient pressure response is revealed by parametric studies. Results show that the injection of polymer increases the peak value of the derivative curve and the flow-continued time, and the surfactant injection affects the pressure transient response in an opposite way to polymer injection. The qualitative explanation for the impacts of polymer and surfactant injection on pressure transient response is given via the changing of mobility in the near wellbore region.

\section{Introduction}

Chemical flooding is one of the most important EOR techniques in which chemical slugs containing polymer, surfactant or alkaline are injected to achieve higher recovery efficiency. The pilot tests conducted in Daqing oil field indicate that the increase of oil recovery by chemical flooding is about $20 \%$ OOIP (Original Oil in Place) which proves the great economic significance of this technique ${ }^{[1]}$.

During the oil production, large amount of transient pressure data is obtained from well test measurements. Yet, conventional well test models and interpretation methodologies do not work for EOR reservoirs and suitable physicochemical models are required to describe the mechanisms of displacement and other key issues affecting the performance of chemical flooding. In the past few decades, a considerable amount of researches have focused on such problems. The pioneering work on the well testing analysis for the polymer flooding was conducted by Ikoku et.al ${ }^{[2]}$ who proposed mathematical models for flow of non-Newtonian power-law fluid in homogeneous porous medium and introduced methods of well test analysis for non-Newtonian fluids. Recently, Escobar et.al ${ }^{[3]}$ applied the TDS (Tiab's direct synthesis) technique to pseudoplastic and dilatants fluids in a radial composite reservoir. Van den Hoek et. al. ${ }^{[4]}$ presented a numerical-analytical combined method of well test interpretation to infer the polymer in-situ rheology from PFO(Pressure Fall-Off) test. For the modeling of more complicated EOR process participated by surfactant, pioneering research was performed by researchers from the university of Texas at Austin. They proposed and improved model for the ASP (alkaline-surfactant-polymer) flooding, and developed a specialized reservoir simulator named $\mathrm{UTCHEM}^{[5]}$. Their works are remarkable for the UTCHEM simulator includes complete descriptions of physicochemical phenomena in chemical flooding which is useful for other researchers for further studies of EOR simulation, including developing numerical simulator for well test analysis. Although much effort has been made since the 1970s, there is still no mature well testing model for polymer-surfactant flooding, and neither practical interpretation methodology applicable to EOR reservoirs. The lack of well testing simulators and interpretation techniques has impeded the formation evaluation and process design in chemical flooding production. 
In this work, a two-phase five-component well test model for polymer-surfactant flooding is established by considering polymer in-situ viscosity, permeability reduction, inaccessible pore volume, adsorption, phase behavior, capillary desaturation, and relative permeability. Based on PEBI gridding, a fully implicit scheme is utilized to the numerical solution of the mathematical model. A substitution of variables method is taken to decrease the number of primary variables of the numerical model. The linear matrix is solved by a GMRES algorithm. Finally, parametric studies are performed which quantify the effect of polymer and surfactant injection on the pressure transient response.

\section{Methodology}

Basic Equations. The mass balance equations are given for each component:

$$
\begin{aligned}
& \nabla\left[\frac{K K_{r o}}{\mu_{o} B_{o}}\left(\nabla p_{o}-g_{o} \nabla D\right)+\frac{K K_{r m} C_{o m}}{R_{k} \mu_{m} B_{m}}\left(\nabla p_{m}-g_{m} \nabla D\right)\right]+q_{o}+q_{m} C_{o m}=\frac{\partial}{\partial t}\left(\frac{\phi S_{o}}{B_{o}}+\frac{\phi_{p} S_{m} C_{o m}}{B_{m}}\right) \\
& \nabla\left[\frac{K K_{r m}}{R_{k} \mu_{m} B_{m}}\left(\nabla p_{m}-g_{m} \nabla D\right) C_{c m}\right]+q_{m} C_{c m}=\frac{\partial}{\partial t} \frac{\phi_{p}\left(S_{m} C_{p m}+C_{a d s, c}\right)}{B_{m}}
\end{aligned}
$$

where $\mathrm{C}_{\mathrm{cm}}$ denotes the concentration of component $\mathrm{c}$ in the microemulsion phase, $c=o, w, p, s, b$ representing the solubilized oil, pure water, polymer, surfactant, and salt.

Polymer in-situ viscosity. In chemical flooding, polymers slugs are injected to achieve a better mobility ratio and a larger swept volume. Therefore, a suitable model should be built to determine the in-situ viscosity of polymer solution. In our simulator, the polymer solution viscosity is calculated in two steps: firstly, the unsheared water phase viscosity $\mu 0$ is determined according to the polymer concentration and salinity using the Flory-Huggins equation:

$$
\mu_{p}=\mu_{w}\left[1+\left(A_{p 1} C_{p}+A_{p 2} C_{p}^{2}+A_{p 3} C_{p}^{3}\right) C_{b}^{S_{p}}\right]
$$

secondly, the non-Newtonian apparent viscosity is calculated by:

$$
\mu_{a p}=\mu_{w}+M \sqsubset\left(\mu_{0}-\mu_{w}\right)
$$

where $\mathrm{M}$ is a shear thinning multiplier, and an experimental $\mathrm{M}$-velocity curve is needed to determine the shear tinning behavior of the polymer solution.

Phase behavior. There are generally three phase environments depending on the sanility. In this work, the Type(II-) phase state is considered. Fig. 1 gives the ternary phase diagram for the Type(II-) phase environment. In the diagram, three apexes of the triangle stand for water $(\mathrm{W})$, surfactant $(\mathrm{S})$ and oil $(\mathrm{O})$, respectively. A binodal curve separates the single phase and two-phase regions. Above the binodal curve is the single phase region and below is the two-phase region. There are a series of tie lines in the two-phase region. The two endpoints of each tie line represent the compositions of the two phases in equilibrium. In this work, we make assumption that the oil phase only contains pure oil. Therefore the right ends of all tie lines coincide with the oil apex. The left end of a tie line represents the microemulsion phase which contains water, surfactant and solubilized oil. The concentrations (volume fractions) of each pseudocomponent in the microemulsion could be calculated by Hand's equation:

$$
C_{s m} / C_{o m}=A_{H}\left(C_{s m} / C_{w m}\right)^{B}
$$

Substitution of Variables Method. The phase behavior of a Type(II-) phase environment could be described by the ternary phase diagram in Figure 1. Two different phase status are possible based on the total composition of oil, water and surfactant. If the composition is located in the two-phase region of the ternary diagram, oil-microemulsion system exists. In this situation, $\mathrm{S}_{\mathrm{m}}$ is a variable and $\mathrm{C}_{\mathrm{om}}$ can be obtained from Eq. 5. If the composition comes into the single-phase region of the phase diagram, the 
equilibrium system will be a single microemulsion phase. In this situation, Eq. 5 is invalid and $\mathrm{C}_{\mathrm{om}}$ should be solved implicitly, meanwhile Sm becomes a constant.

In general, we have two sets of primary variables, which are $\left(p_{o}, p_{w f}, S_{m}, C_{p m}, C_{s m}, C_{b m}\right)$ for the single phase situation and $\left(\mathrm{p}_{\mathrm{o}}, \mathrm{p}_{\mathrm{wf}}, \mathrm{C}_{\mathrm{om}}, \mathrm{C}_{\mathrm{pm}}, \mathrm{C}_{\mathrm{sm}}, \mathrm{C}_{\mathrm{bm}}\right)$ for the two-phase situation. As the two situations are exclusive, a substitution of variables method could be applied here which generates separate forms of expansion for different phase state changings in a times step, and thus decrease the number of primary variables.

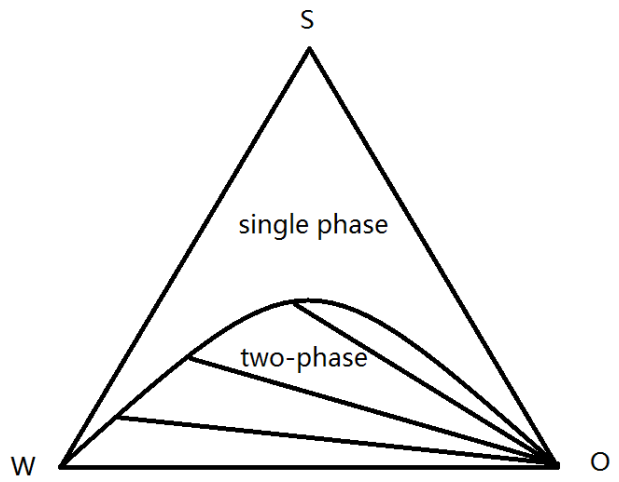

Figure 1. Type(II-) ternary phase diagrams

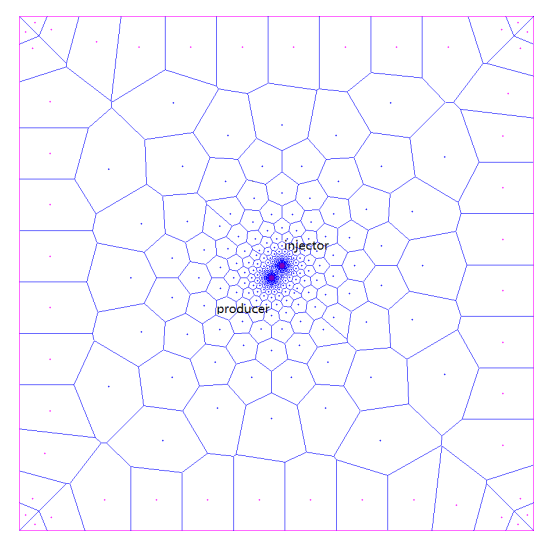

Figure 2. Calculation region and gridding

\section{Results And Discussion}

In this part, we study on a $4000 \times 4000 \times 10 \mathrm{~m}$ single-layer homogeneous reservoir. Two wells are located in the reservoir, as shown in Fig. 2. The production well produces at a constant liquid rate of 10 $\mathrm{m}^{3} /$ day at surface condition. The injection well injects polymer solution at a constant liquid rate of 10 $\mathrm{m}^{3} /$ day at surface condition for 300 days and then shuts in for 100 days. Reservoirs parameters for calculation are listed in Table 1. Numerical simulation is performed and the BHP (bottom hole flowing pressure) values of the injection well in the pressure fall-off regime are calculated which are given in Fig. 3 and Fig. 4.

Table 1 Input parameters

\begin{tabular}{|c|c|c|c|}
\hline Initial reservoir pressure, $\mathrm{MPa}$ & 20 & compressibility of oil, $1 / \mathrm{MPa}$ & 0.006 \\
\hline $\begin{array}{c}\text { Initial water saturation, } \\
\text { dimensionless }\end{array}$ & 0.6 & compressibility of water, $1 / \mathrm{MPa}$ & 0.002 \\
\hline Layer thickness, $\mathrm{m}$ & 10 & viscosity of oil, $\mathrm{Pa} \cdot \mathrm{s}$ & 0.004 \\
\hline Horizontal permeability, $\mu \mathrm{m}^{2}$ & 0.2 & viscosity of pure water, $\mathrm{Pa} \cdot \mathrm{s}$ & 0.001 \\
\hline Porosity, fraction & 0.2 & well bore radius, $\mathrm{m}$ & 0.1 \\
\hline reference pressure, MPa & 20 & Skin factor, dimensionless & 0 \\
\hline compressibility of rock, 1/MPa & 0.00015 & Oil-water IFT ${ }^{\text {om }}, \mathrm{N} / \mathrm{m}$ & 0.02 \\
\hline $\begin{array}{c}\text { parameters for calculation of } \\
\text { polymer viscosity Ap1 Ap2 Ap3 Sp }\end{array}$ & $\begin{array}{c}81,2700, \\
2500,0.17\end{array}$ & $\begin{array}{c}\text { Interfacial tensor parameters CIFT, } \\
a\end{array}$ & $0.3,10$ \\
\hline
\end{tabular}

Fig. 3 gives the log-log plot of the pressure change and pressure derivative for different injection polymer concentrations. The figure shows that as the polymer concentration increases, the pressure drop becomes lager and the peak value of the derivative also increases. The reason is that the increase of polymer concentration reduces the fluid mobility near the wellbore and thus leads to a larger pressure rise during the injection period. Therefore, after the well shuts in, the pressure drop is also larger. As the mobility is reduced, the flow-continued time of the wellbore storage and transition regime becomes longer. In the late period, the curves coincide with each other because the mobility in regions far from the wellbore is not changed as the polymer front does not reach.

Fig. 4 gives the log-log plot of the pressure change and pressure derivative for various surfactant concentrations. It is observed that when surfactant is injected the pressure drop becomes smaller comparing to the water flooding case $(\mathrm{Cs}=0)$, and the peak value of the derivative becomes lower. The 
higher the surfactant concentration is, the smaller the pressure drop and the peak value becomes. The reason is that with the flooding of surfactant slug, the mobility of fluids in the near well bore region is improved. Therefore, the pressure drop of the PFO stage becomes smaller and the peak value of the derivative is lower. The flow-continued time of the wellbore and transition stage is also shorter as the mobility increases. In the late period, derivative curves for different surfactant concentrations coincide with each other, because the mobility in regions far from the wellbore is not changed as the surfactant slug does not reach.

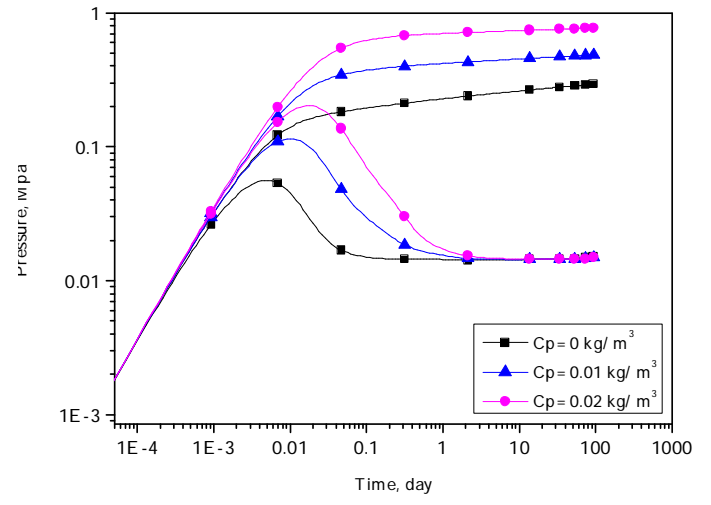

Figure 3. Well bottom pressure and pressure derivative of the pressure fall-off period for various polymer concentration

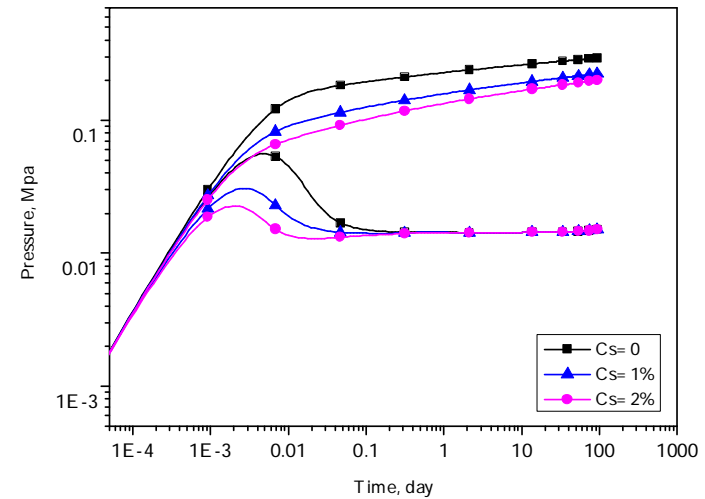

Figure 4. Well bottom pressure and pressure derivative of the pressure fall-off period for various injection polymer concentrations

\section{Summary}

In this paper, a two-phase five-component well test model for polymer-surfactant flooding is proposed considering the in-situ viscosity of polymer, phase behavior of microemulsion and other key issues in the flooding process. Based on PEBI gridding, a fully implicit numerical well test simulator is developed.

By conducting parametric studies through a PFO test example, we find that the polymer and surfactant concentration greatly affects the pressure transient response. The increase of polymer concentration will leads to a higher peak value of the derivative curve and a longer wellbore storage and transition regime. The surfactant concentration affects the pressure transient response in an opposite way. The pressure transient response can be explained by considering the change of mobility in the near wellbore region after polymer and surfactant injection.

\section{References}

[1]Wang, D, Cheng, J., Wu, J., Yang, Z. and Yao, Y., (1999). Summary of ASP Pilots in Daqing Oil Field. paper SPE 57288 presented at the 1999 Asia Pacific Improved Oil Recovery Conference, Kuala Lumpur, Malaysia, Oct 25-26.

[2]Ikoku, ChiU.; Ramey Jr., Henry J. 1979. Transient Flow of Non-Newtonian Power-Law Fluids in Porous Medium. SPE J 19 (3):164-174. SPE 7139-PA.

[3]Escobar, F.H., Martinez, J.A., and Montealegre-M., Matilde, 2010. Pressure and Pressure Derivative Analysis for a Well in a Radial Composite Reservoir with a Non-Newtonian /Newtonian Interface. CT\&F. 4 (1): 33-42.

[4]Van den Hoek, P., Mahani, H., Sorop, T., Brooks, D., Zwaan, M., Sen, S., \& Saadi, F. (2012, January). Application of Injection Fall-Off Analysis in Polymer flooding. In SPE Europec/EAGE Annual Conference. Society of Petroleum Engineers.

[5]Pope, G.A., Nelson, R.C., (1978). A Chemical Flooding Compositional Simulator. Society of Petroleum Engineers Journal 18, 5, 339 - 354. 